\title{
AVALIAÇÃO DOS CONHECIMENTOS TECNOLÓGICOS, PEDAGÓGICOS E DISCIPLINARES NO ENSINO DA CIÊNCIA
}

\author{
EVALUACIÓN DE LOS CONOCIMIENTOS TECNOLÓGICOS, \\ PEDAGÓGICOS Y DISCIPLINARES EN LA ENSEÑANZA DE LA CIENCIA
}

\author{
EVALUATION OF PEDAGOGICAL, DISCIPLINARY AND \\ TECHNOLOGICAL KNOWLEDGE IN SCIENCE TEACHING
}

\author{
Brenda Luz COLORADO-AGUILAR ${ }^{1}$ \\ Berenice MORALES-GONZÁLEZ ${ }^{2}$
}

RESUMO: O presente trabalho se trata de uma pesquisa em andamento focada na avaliação dos conhecimentos pedagógicos, disciplinares e tecnológicos no ensino da ciência em cursos de ensino pré-escolar e ensino fundamental da Escola Normal Veracruzana "Enrique C. Rébsamen" em Xalapa, Veracruz, México. O estudo é proposto no contexto do modelo "Technological, Pedagogical, Content Knowlege" (TPACK) desenvolvido por Mishra e Koehler (2006) e Koehler e Mishra (2008), com base na análise de conhecimento Pedagogical Content Knowledge (PCK) de Shulman (1986; 1987). Este esquema se baseia na inter-relação entre os componentes pedagógicos, disciplinares e tecnológicos para a formação de professores no ensino da ciência. Espera-se que os resultados permitam obter informações rigorosas sobre o alcance do perfil docente em relação ao ensino das ciências naturais e, sobretudo, divulgar um paradigma atualizado do ensino da ciência a partir da formação inicial de professores.

PALAVRAS-CHAVE: Formação inicial de professores. Ensino da ciência. Tecnologia educacional.

RESUMEN: Se presenta una investigación en curso enfocada en la evaluación de los conocimientos pedagógicos, disciplinares y tecnológicos en la enseñanza de la ciencia en las licenciaturas en educación preescolar, primaria y especial de la Escuela Normal Veracruzana "Enrique C. Rébsamen" en Xalapa, Veracruz, México. El estudio se propone desde una perspectiva epistemológica que estudia la inserción de la tecnología en el aula de una forma integral y sistémica, en el marco del modelo Technological, Pedagogical, Content Knowledge (TPACK) elaborado por Mishra y Koehler (2006) y

Koehler y Mishra (2008), basado en el análisis del constructo Conocimiento Pedagógico del Contenido (PCK) de Shulman (1986; 1987). Dicho esquema fundamenta la interrelación entre los componentes pedagógicos, disciplinares y tecnológicos para la

\footnotetext{
${ }^{1}$ Benemérita Escuela Normal Veracruzana “Enrique C. Rébsamen”. Xalapa - México. Doctora en Sistemas y ambientes educativos. ORCID: <http://orcid.org/0000-0002-1978-5317> E-mail: brendaluzcolorado@gmail.com

${ }^{2}$ Benemérita Escuela Normal Veracruzana “Enrique C. Rébsamen”. Xalapa - México. Doctora en Sistemas y ambientes Educativos. ORCID: <http://orcid.org/0000-0002-0690-5324>. E-mail: berenice_mg@yahoo.com
} 
formación de docentes en la enseñanza de la ciencia. Se espera que los resultados permitan obtener información rigurosa sobre el alcance del perfil docente con relación a la enseñanza las ciencias naturales y sobretodo difundir un paradigma actualizado de la enseñanza de la ciencia desde la formación inicial docente.

PALABRAS-CLAVE: Formación inicial docente. Enseñanza de las ciencias. Tecnología educativa.

ABSTRACT: This papers describes a research proyect focused on the evaluation of technological, pedagogical and content knowledge in science teaching in the preschool, primary and special education degrees at the Escuela Normal Veracruzana "Enrique C. Rébsamen" in Xalapa, Veracruz, México. The study is proposed from an epistemological perspective that studies the insertion of technology in the classroom from an integral and systemic way: the framework of the Technological, Pedagogical, Content Knowledge (TPACK) model developed by Mishra and Koehler (2006) and Koehler and Mishra (2008), based on the analysis of the construct Pedagogical Content Knowledge (PCK) proposed by Shulman (1986; 1987). This scheme is the basis for understanding the interrelation between the pedagogical, disciplinary and technological components on teachers training for science teaching. It is expected that the results allow to obtain rigorous information about the scope of teaching profile in relation to the natural sciences teaching and spreading an innovative paradigm of the science teaching from the initial teacher training.

KEYWORDS: Initial teacher training. Science teaching. Educacional technology

\section{Introdução}

[...] as crianças, desde pequenas, vão construindo teorias explicativas da realidade de um modo similar ao que utilizam os cientistas... fazer ciência não é conhecer a verdade, mas tentar conhecê-la. Por tanto, devemos propiciar às crianças uma atitude de pesquisa que se fundamente nos critérios da relatividade e não em critérios dogmáticos [...] (TONUCCI, 1995, p. 85).

A formação inicial, do mesmo modo que a formação continuada, se situam como foco para o alcance dos objetivos do modelo educativo mexicano 2017. A partir desse modelo, espera-se do professor não apenas o domínio disciplinar e pedagógico, como também o desenvolvimento da capacidade de aprender a aprender, o desenho de ambientes de aprendizagem efetivos e inclusivos, o desenvolvimento de projetos educativos com uma visão integradora, o uso pedagógico das tecnologias de informação e comunicação (TIC) para a criação de ambientes de aprendizagem que apoiem ao desenvolvimento de conhecimentos, habilidades, atitudes e valores. Em suma, se conceitua o docente como um 
"profissional centrado na aprendizagem de seus alunos, que gera ambientes de aprendizagem e inclusão, comprometido com a melhoria constante de sua prática docente e capaz de adaptar o currículo ao contexto específico" (SEP, 2017, p. 30). Um foco que se visualizava já desde o Plano de Estudos 2011, tendo em vista a construção de "uma escola mexicana que responda às demandas do século XXI” SEP, 2011, p. 17). Estas exigências, ainda não resolvidas, mantêm desafios para a formação inicial quanto a promover a plena participação dos estudantes normalistas, docentes formadores e autoridades educativas na transformação das escolas normais a instituições de educação superior de qualidade suficiente para oferecer uma profissão inovadores e congruente às exigências sociais atuais e futuras.

Embora a incorporação das TIC no planejamento e implementação da formação docente é condição obrigatória nos mais recentes programas a nível internacional, vide o caso do novo currículo australiano (SHEFFIELD; DOBOZY; GIBSON; MULLANEY; CAMPBELL, 2015), seu domínio didático especificamente para o ensino da ciência resulta imprescindível. Não bastam os conhecimentos tecnológico, conhecimento pedagógico e conhecimento disciplinar para contribuir desde a formação docente até o perfil desenhado através do novo modelo educativo 2017. O anterior implica abordar a articulação entre o conteúdo, a didática e o conhecimento sobre os recursos a serem empregados nas atividades com os estudantes para gerar espaços efetivos de aprendizagem.

Em nosso país, o ensino da ciência e da tecnologia tem, de acordo com Flores (2012), baixo investimento nesses setores, assim como reduzida presença como parte da cultura nacional, ainda que no discurso institucional se ressalte sua importância para o desenvolvimento, o país ressalta a ciência, a tecnologia e a inovação como elementos fundamentais dentro do novo Modelo Educativo em nível básico (SEP, 2016a).

Neste sentido, o ensino das ciências em educação básica representa uma via investigativa a ser explorada através da formação inicial docente. Por isso, o objetivo desse trabalho é apresentar um projeto de investigação que avalie a articulação dos conhecimentos pedagógicos, disciplinares e tecnológicos para o ensino das ciências na formação inicial docente. Interessa, então, explorar os conhecimentos através de um paradigma sistêmico que considere a dimensão tecnológica, pedagógica e disciplinar das ciências por meio da percepção de docentes em formação e seus professores. De igual modo, interessa analisar essas percepções e sua contribuição no ensino das ciências. Dentro da formação em Escolas Normais do Estado de Veracruz, é o primeiro projeto aprovado e 
financiado pelo Fundo Setorial Instituto Nacional para a Avaliação da Educação (INEE) e pelo Conselho Nacional de Ciência e Tecnologia (CONACyT).

O estudo se propõe no marco do modelo Technological, Pedagogical, Content Knowledge (TPACK) elaborado por Mishra y Koehler (2006) y Koehler y Mishra (2008), baseado na análise do construto Conhecimento Didático do Conteúdo (PCK) de Shulman (1986; 1987). O modelo TPACK representa um modelo que apoia a integração da tecnologia em sua prática educativa através de três tipos de conhecimentos: pedagógicos, de conhecimento ou disciplinar e tecnológicos (CABERO, 2014).

Como estudo misto, se considera uma fase quantitativa e outra qualitativa. A partir do enfoque quantitativo se confirmará ou não as seguintes hipóteses do trabalho: 1) A maior percepção de domínio dos conhecimentos tecnológicos e de conteúdo dos docentes em formação, maior contribuição no desenho, desenvolvimento e avaliação de situações didáticas em ciências; e 2) A percepção de conhecimentos TPACK dos professores se relaciona com a percepção identificada por seus alunos. A população participante deste estudo se estabelece em cursos de graduações em educação primaria, pré-escolar e especial, em duas escolas formadoras de docentes do estado de Veracruz, México.

\section{Histórico}

Até o século passado, o ensino das ciências naturais se vinculava à formação de estudantes para a realização de estudos científicos pelo qual apenas uma minoria chegava a estudar nestas áreas. Atualmente, se considera que ensinar ciências nos níveis de educação obrigatória é de suma importância para a aquisição de competências, habilidades, valores y aptidões para melhorar a qualidade de vida das pessoas y dar mais possibilidades de interagir no mundo e na sociedade (INEE, 2017).

Para visualizar o papel da disciplina de ciência dentro do nosso sistema educativo comparado com outros países, compartilha-se um estudo realizado pelo INEE (2017), no qual se analisou o currículo de cinco países que foram selecionados conforme os seguintes critérios:

- Qualidades do país, segundo a porcentagem do produto interno bruto (PIB) que cada país dedica à educação, população e índice de desenvolvimento.

- Características do currículo de cada país, segundo resultado obtido em 
provas internacionais, acesso e idioma de documentos curriculares.

Os resultados obtidos no referido estudo se mostram na tabela 1, na qual se evidencia as diferenças em relação aos momentos de ensino das ciências naturais nos diferentes níveis educativos dos países estudados. Em nosso país, assim como na Holanda, a educação em ciências inicia aos três anos, diferentemente dos demais países.

Tabela 1: Comparação de disciplinadas relacionadas à ciência em diferentes países

\begin{tabular}{|c|c|c|c|c|c|c|}
\hline $\begin{array}{l}\text { Idade } \\
\text { de } \\
\text { Ingreso }\end{array}$ & Brasil & Chile & $\begin{array}{l}\text { Corea do } \\
\text { Sul }\end{array}$ & $\begin{array}{l}\text { Estados } \\
\text { Unidos }\end{array}$ & Holanda & México \\
\hline 3 & & & & & & Exploração e \\
\hline 4 & & & & & & conhecimento \\
\hline 5 & & & & & Orientação & $\begin{array}{l}\text { do mundo } \\
\text { natural. }\end{array}$ \\
\hline 6 & \multirow{9}{*}{$\begin{array}{l}\text { Ciências } \\
\text { Naturais }\end{array}$} & \multirow{8}{*}{$\begin{array}{l}\text { Ciências } \\
\text { da vida, } \\
\text { física, } \\
\text { química } \\
\text { da Terra e } \\
\text { do } \\
\text { Universo. }\end{array}$} & & $\begin{array}{l}\text { Ciência: a } \\
\text { natureza da } \\
\text { ciência, a }\end{array}$ & $\begin{array}{l}\text { pessoal e no } \\
\text { Mundo } \\
\text { Natureza e }\end{array}$ & $\begin{array}{l}\text { Exploração da } \\
\text { natureza e da } \\
\text { sociedade. }\end{array}$ \\
\hline 7 & & & & $\begin{array}{l}\text { natureza da } \\
\text { matemática, a }\end{array}$ & Tecnologia. & \\
\hline 8 & & & \multirow{8}{*}{$\begin{array}{l}\text { Ciência: } \\
\text { movimento e } \\
\text { energia, } \\
\text { materiais, } \\
\text { vida e terra e } \\
\text { espaço. }\end{array}$} & natureza da & & \multirow{4}{*}{$\begin{array}{l}\text { Ciências } \\
\text { naturais }\end{array}$} \\
\hline 9 & & & & tecnologia, o & & \\
\hline 10 & & & & entorno físico, & & \\
\hline 11 & & & & vivo, o & & \\
\hline 12 & & & & organismo & & Biologia \\
\hline 13 & & & & humano, & Homem e & Física \\
\hline 14 & & \multirow{2}{*}{$\begin{array}{l}\text { Biologia, } \\
\text { Física e } \\
\text { Química }\end{array}$} & & sociedade & & Química \\
\hline 15 & \multirow{3}{*}{$\begin{array}{l}\text { Ciências } \\
\text { Naturais: } \\
\text { Biologia, } \\
\text { Física e } \\
\text { Química }\end{array}$} & & & $\begin{array}{l}\text { humana, o } \\
\text { mundo }\end{array}$ & & \multirow{3}{*}{$\begin{array}{l}\text { Biologia, } \\
\text { Física, } \\
\text { Química, etc. }\end{array}$} \\
\hline 16 & & & & $\begin{array}{l}\text { desenhado, o } \\
\text { mundo }\end{array}$ & & \\
\hline 17 & & & & $\begin{array}{l}\text { matemático. } \\
\text { Perspectivas } \\
\text { históricas, } \\
\text { temas comuns, } \\
\text { hábitos da } \\
\text { mente. }\end{array}$ & & \\
\hline
\end{tabular}

*Nota: Reprodução do Estudo comparativo da proposta curricular de ciências em educação obrigatória no México e outros países, segundo INEE (2017, p. 34).

\section{Contexto}

Dentro da formação de professores de nível básico no contexto mexicano, o plano de estudos vigente das graduações em Educação Primária e Pré-escolar, sustenta a busca de um profissional graduado que desenvolva um pensamento científico e uma visão integral do fenômeno educativo para a reflexão, investigação e resolução permanente de problemas, ou seja, aspira a "formar um docente de educação básica que utilize 
argumentos científicos, pedagógicos, metodológicos, técnicos e instrumentais para entender y fazer frente às complexas exigências que a docência impõe” (SEP, 2012).

Do mesmo modo, nos cursos de graduação em Educação Especial, o plano de estudos vigente que data o ano de 2004, aponta que se deve fomentar o interesse e a curiosidade científica dos normalistas, aproximando-os das diversas noções e práticas próprias do pensamento científico, para alcançar o uso crítico e analítico tanto da informação - produto da investigação, como de critérios e instrumentos adequados.

O plano de estudos de graduação em Educação Primária se localiza no curso de Ciências Naturais (2013) onde se busca a construção e a concepção da ciência, considerada competência científica como capacidade de um indivíduo que tem conhecimento científico, o que permite a mobilização de sabres múltiplo e atitudes científicas como saber de e sobre a ciência, o saber fazer ciência e o saber ser em diferentes contextos e cenários, um curso que, embora aponte para o desenho de propostas didáticas, se baseia na construção de noções sobre o que é ciência, como se adquire, se valida, se ensina e se aprende (SEP, 2013). No caso da graduação pré-escolar 2012, se identifica no curso Aproximação das ciências naturais na pré-escola o interesse no desenvolvimento integral do futuro docente através da compreensão da ciência (SEP, 2012). Pareceria se delinear a ênfase de uma formação próxima da ciência através de determinadas disciplinas. No caso da graduação em educação especial, se encontra, de maneira velada, um interesse pelo desenvolvimento de disposição, capacidades e atitudes próprias da investigação científica, a partir dos traços do perfil do egresso, não se encontrou uma disciplina diretamente relacionada ao ensino da ciência. Desde o ciclo escolar 2011 - 2012, identificou-se a integração de 100 serviços de educação especial ao Programa de Aplicação dos Sistemas de Ensino Vivencial e indagatória das Ciências PASEVIS, criado desde 2006 no estado de Veracruz como um programa dirigido aos serviços de educação de nível básico, iniciativa do governo federal por condução da Secretaria de Educação de Veracruz em colaboração com a associação Inovação no Ensino e na Ciência (INNOVEC).

O corpo académico de Políticas Públicas e Avaliação Educativa da Benemérita Escolar Normal Veracruzana "Enrique C. Rébsamen" (BENV) tem investigado as percepções dos estudantes sobre o papel da ciência e da tecnologia, a investigação básica, os cientistas e o governo (ZÁRATE, MORALES, PÉREZ, 2016). Para isso, aplicou-se a 345 alunos regulares, um instrumento validado e amplamente utilizado pelo Conselho 
Nacional de Ciência e Tecnologia (CONACYT), em colaboração com o Instituto Nacional de Estatística e Geografia (INEGI), no marco da Pesquisa sobre a Percepção Pública da Ciência e Tecnologia (ENPECYT), durante os anos 1997, 2001, 2003, 2005, 2007, 2009 y 2013

Um dado relevante levantado nesse estudo identifica que $30 \%$ dos estudantes não concordam que a ciência e a tecnologia fazem nossas vidas mais fáceis, confortáveis e saudáveis; ao lado desde dado, $74.5 \%$ dos estudantes não concordam que ajudarão a erradicar a pobreza e a fome. O que implica que, em relação à percepção social da ciência em estudantes da BENV, é importante fazer chegar o ensino das ciências a todos os alunos como algo útil e relevante, tal como menciona Acevedo (2004). O ensino da ciência com a vida real, que apoie a formação de um espírito crítico e questionador de dogmas, que possa diferencias a evidência empírica da opinião pessoal (GIL; VILCHES, 2006).

\section{Fundamento teórico}

Há esforços investigativos na região para transformar as práticas cotidianas do ensino das ciências na educação básica por meio de:

- A integração pedagógica de ferramentas tecnológicas de um estilo de ensino que promova aprendizagens significativas em ciências. (DORIA; ZERMEÑO; ARREDONDO, 2014; MACÍAS, 2011);

- O desenvolvimento de sistemas tecnológicos para modernizar e apoiar o ensino das ciências através de plataformas e ambientes de aprendizagem de trabalho virtual. (SÁNCHEZ, 2013; PÓSITO, 2012; MORENO; PINTOR; GÓMEZ, 2016);

- O desenvolvimento de recursos digitais como livros interativos multimídia para o processo de ensino das ciências naturais. (VASCO; ACURIO, 2015).

Os cenários atuais, onde se desenvolve o processo educativo, representam um fator fundamental para o desenvolvimento de projetos, nos quais se propõe a incorporação tecnológica de acordo com as características de cada entorno como: percepção e formação nas TIC; competência digital dos professores; capacidade para interagir e fazer uso das potencialidades e características as TIC, bem como das características cognitivas e sociais dos estudantes. 
Cabero (2014) considera que a percepção que têm os docentes do uso das TIC e do grau de formação a respeito da sua utilização é significativa para sua incorporação em sala de aula. Neste sentido, é importante analisar o que os docentes são capazes de fazer com elas, bem como sua criatividade para interagir com as novas formas de comunicação, a forma de resolver problemáticas educativas de maneira inovadora e as características sociais e cognitivas de seus estudantes.

Neste contexto, para Bonil (2009) é importante considerar que "os professores devem desenvolver um conhecimento da matéria, um conhecimento psicopedagógico geral, um conhecimento de como ensinar a matéria específica” (p. 449). Isso implica a plena articulação entre o conteúdo, a didática e o conhecimento sobre os recursos a empregar nas atividades com os estudantes para gerar espaços efetivos de aprendizagem.

Esta área de oportunidade é considera uma via para investigar não só os conhecimentos tecnológicos com os quais os docentes conta, mas também, a partir de um olhar integral, identificar a dimensão didática pela qual cada docente compreende e aplica seus conhecimentos tecnológicos para favorecer à aprendizagem contextualizada em cada curso. O modelo TPACK, se apresenta como um modelo integral-sistêmico que possa exceder certo determinismo tecnológico ou pedagógico (CABERO, 2014).

Embora existam iniciativas de modelos conceituais para o uso e incorporação das TIC no processo educativo, o modelo TPACK tem sido reconhecido por diferentes autores, como um esquema de maneira estruturada e sistêmica. Ying-Shao, Yi-Fen y Hsin-Kai (2015) realizaram um estudo no qual obtiveram como produto final um modelo de trabalho dirigido a docentes e baseado no modelo TPACK para gerar novas ideias com o uso da tecnologia no ensino da ciência. No referido estudo, se capacitou os docentes sobre aspectos que deveriam contemplar os programas para formação de docentes. Autores como Abbitt (2011) y Bilici, Yamak, Kavak y Guzey (2013), realizaram estudos acerca das crenças de auto eficácias sobre tecnologia a partir do modelo TPACK, considerando a vinculação que existe entre essas premissas.

Koehler y Mishra, (2009) así como Koehler, Mishra, y Cain (2013) desenharam o modelo Technological, Pedagogical Content Knowledge denominado TPACK, ou modelo de Conhecimento Tecnológico, Pedagógico e de Conteúdo, baseado no modelo Pedagogical Content Knowledge (PCK) de Shulman (1986), a aprovação dos autores são os elementos de Tecnologia (T), Pedagogia (P) e Conteúdo curricular (C), bem como as 
interações em pares e tríada sucedida entre esses componentes, o que dá lugar a novos componentes (ver figura 1).

Figura 1: Modelo TPACK

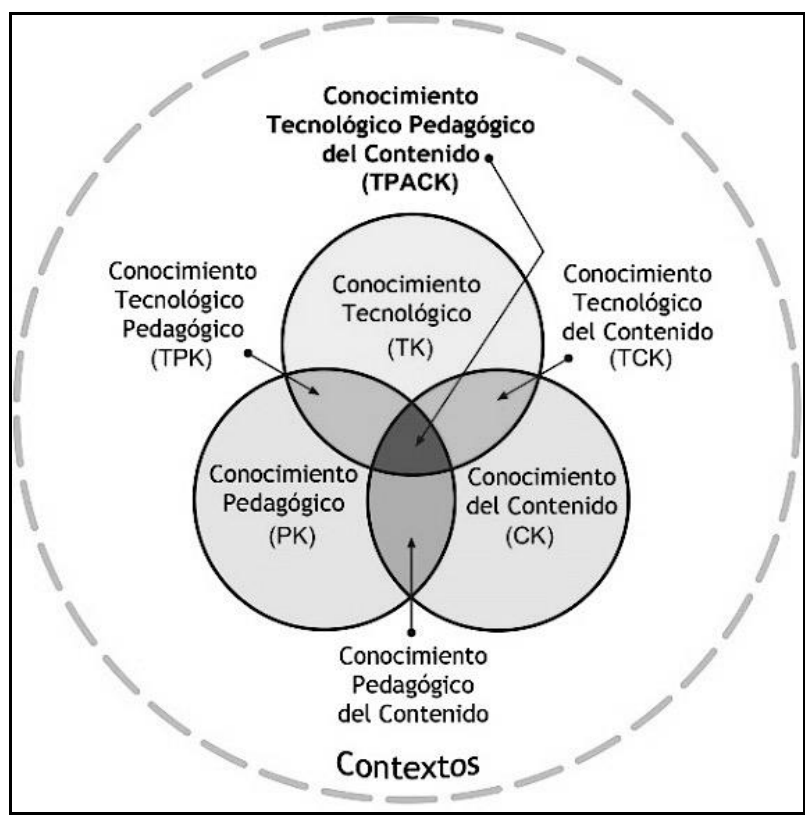

Fonte: Koehler (2012)

O planejamento de Shulman (1986) é a essência e ponto de partida do modelo TPACK. Ele destaca a reflexão sobre os conhecimentos (tecnológicos, pedagógicos e de conteúdo, ou disciplinar) necessários a todo professor para incorporar de forma eficaz às ferramentas tecnológicas em sus afazeres docentes. A compreensão de cada um desses campos de conhecimento não é suficiente. É necessário um aprofundamento sobre as interações entre estes três conhecimentos.

Esta visão multidimensional demanda do trabalho docente uma intervenção que ultrapasse o trabalho isolado e implique na integração de equipes interdisciplinares em pedagogia, tecnologia e áreas de diversas disciplinas, ou seja, se "exige uma reformulação na formação de professores e práticas atuais" (VALVERDE et al, 2010, p. 204). Esta premissa, considerada como base de boas práticas educativas com TIC supera "a falsa dicotomia entre pedagogia e tecnologia" (p. 210) entre os conhecedores das tecnologias de aprendizagem, das realidades educativas em sala de aula, resistentes à mudança e visão dos tecnólogos como técnicos geradores de propostas superficiais.

\section{Metodologia}

RIAEE - Revista Ibero-Americana de Estudos em Educação, Araraquara, v. 13, n. 03, p. 997-1010, jul./set., 2018. E-ISSN: 1982-5587. 
A investigação a realizar se aplica e de estudo ex-post-facto encontra-se no tipo misto (HERNÁNDEZ; FERNÁNDEZ; BAPTISTA, 2010) que permite um estudo mais profundo de um tema cuja complexidade baseada na utilização de somente um dos enfoques. O objetivo geral busca avaliar a articulação dos conhecimentos pedagógicos, disciplinares e tecnológicos para o ensino das ciências na formação inicial docente. Na tabela 2 se especifica os cursos considerados nesse estudo, e os participantes professores e alunos regulares:

Tabla 2: Participantes da pesquisa

\begin{tabular}{|c|c|c|}
\hline $\begin{array}{l}\text { Graduação } \\
\text { Primária }\end{array}$ & $\begin{array}{l}\text { Graduação em Educação Pré- } \\
\text { escolar }\end{array}$ & $\begin{array}{l}\text { Graducação em } \\
\text { Educação Especial }\end{array}$ \\
\hline 20 cursos e alunos. & 18 cursos e alunos & 4 cursos \\
\hline $\begin{array}{l}* 4 \text { cursos da trajetória } \\
\text { formativa Preparação do Ensino } \\
\text { e Aprendizagem centrados nos } \\
\text { conhecimentos disciplinares da } \\
\text { ciência e seu ensino. }\end{array}$ & $\begin{array}{l}* 3 \text { cursos da trajetória } \\
\text { formativa Preparação do } \\
\text { Ensino e Aprendizagem } \\
\text { centrados nos conhecimentos } \\
\text { disciplinares de ciência e seu } \\
\text { ensino }\end{array}$ & $\begin{array}{c}4 \text { cursos } \\
\text { correspondentes a } \\
\text { atividades de } \\
\text { aproximação à pratica } \\
\text { escolar }\end{array}$ \\
\hline $\begin{array}{c}* 1 \text { curso da trajetória } \\
\text { Língua adicional e Tecnologia } \\
\text { da informação }\end{array}$ & $\begin{array}{c}\text { *1 curso da trajetória } \\
\text { Língua adicional e Tecnologia } \\
\text { da informação }\end{array}$ & \\
\hline $\begin{array}{l}* 5 \text { cursos traj } \\
\text { Prática profissional }\end{array}$ & $\begin{array}{r}* 5 \text { cur } \\
\text { Prática profissi }\end{array}$ & \\
\hline
\end{tabular}

*Nota: O total de cursos é de 84 . O número de estudantes a avaliar se estima entre 2,100 alunos.

Através do paradigma quantitativo, a partir de uma análise dedutiva se busca confirmar as seguintes hipóteses:

1) A percepção de conhecimentos TPACK dos professores se relaciona com a percepção identificada por seus alunos, encontrados em cursos selecionados de segundo a sétimo ano.

2) A maior percepção de domínio dos conhecimentos tecnológicos pedagógicos e de conteúdo, maior contribuição em desenho, desenvolvimento e avaliação de situações didáticas em ciências. 
O instrumento a se utilizar nesta fase corresponde a um questionário tipo Likert, elaborado Schimidt, Baran, Thompson, Mishra, Koehler, y Shin (2009), traduzido e validado por Cabrero (2014) e um questionário complementário que recupere dados demográficos de participantes, no caso de alunos regulares se agregarão perguntas que recuperem sua percepção de contribuição no desenho, desenvolvimento e avaliação de situações didáticas em ciências. Ambos instrumentos permitirem dar uma resposta quantitativa aos dois objetivos deste estudo. Para explorar os diferentes tipos de conhecimentos que possuem tanto professores como estudantes, se fará uso das estatísticas descritivas através do software SPSS, serão determinadas as diferenças de percepções entre participantes através do Teste de contrastes de mídias, provas de comparações múltiplas e provas de correlação para analisar se as percepções dos alunos a respeito dos seus conhecimentos (TPACK) contribuem em sua percepção de desenho, desenvolvimento e avaliação de situações didáticas de ciências.

A partir da perspectiva qualitativa, considera-se epistemologicamente construtivista (GUBA; LINCOLN, 1994), tendendo à compreensão das construções que as pessoas elaboram (incluindo o pesquisador). Foi selecionado o estudo de caso, como método qualitativo, orientado a aprofundar o segundo objetivo: analisar as percepções de estudantes regulares a respeito da formação recebida em conhecimentos disciplinares da ciência, didáticos e tecnológicos (TPACK) e sua contribuição no desenho, desenvolvimento e avaliação de situação em ciências.

Espera-se que os resultados permitam obter informações relevantes sobre o escopo desses traços de perfil docente com relação ao ensino da disciplina de ciências naturais especificamente e, sobretudo, difundir um paradigma atualizado do ensino da ciência através da formação inicial docente que integre o uso pedagógico de ferramentas, segundo as características dos alunos e assuntos disciplinares a representar.

\section{Conclusão}

Em nosso país, grande parte dos docentes e educação básica se formam nas escolas regulares, assumindo as reformas curriculares que vão transformando as propostas de educação. No entanto, de acordo com Robles (2012), atualmente se requer de docentes uma profissionalização constante e vigente para atender a crianças que vivem em inovação. Seres integrais com a necessidade de ter maior conhecimento e valorização da ciência para 
favorecer a resolução de situações da vida cotidiana (INEE, 2017). Por tanto, em nosso país, é importante repensar a atenção em propostas que favoreçam a formação de docentes no campo das ciências, considerando que se desempenam com estudantes que, por natureza, têm interesse de indagação pelas situações à sua volta. Se trata, então, de gerar conhecimentos de gerar conhecimentos que apoiem a alfabetização científica na formação integral dos docentes para potencializar sua capacidade de compreensão, valorização, relação e coincidência do mundo que nos rodeia (INEE, 2017, p. 16).

\section{REFERÊNCIAS}

ABBITT, J. An Investigation of the Relationship between Self-Efficacy Beliefs about Technology Integration and Technological Pedagogical Content Knowledge (TPACK) among Preservice Teachers, Journal of Digital Learning in Teacher Education, v. 27 n. 4, p. 134-143, 2011. DOI: 10.1080/21532974.2011.10784670.

ACEVEDO, J. A. Reflexiones sobre las finalidades de la enseñanza de las ciencias: educación científica para la ciudadanía. Revista Eureka sobre Enseñanza y Divulgación de las Ciencias, n. 1, p. 3-15, 2004.

BILICI, S.; YAMAK, H.; KAVAK, N.; GUZEY, S. Technological pedagogical content knowledge self-efficacy scale (TP ACK-SeS) for pre- service science teachers: Construction, validation and reliability.Egitim Arastirmalari-. Eurasian Journal of Educational Research, n. 52, p. 37-60, 2013.

BONIL, J.; MÁRQUEZ, C. ¿Qué experiencias manifiestan los futuros maestros sobre las clases de ciencias? Implicaciones para su formación. Revista de Educación, n. 354, p. 447-472, 2009.

CABERO, J. La formación del profesorado en TIC: Modelo TPACK (Conocimiento Tecnológico, Pedagógico y de Contenido). Sevilla: Publidisa, 2014. ISBN: 978-84-15881-67-4.

DORIA, C. A. H.; ZERMEÑO, M. G. G.; ARREDONDO, M. B. Inclusión de las tecnologías para facilitar los procesos de enseñanza - aprendizaje en ciencias naturales. Tesis de maestría. México, 2014.

KOEHLER, J. 2012. Altura: 768 pixeles. Largura: 768 pixeles. 167 kb. Formato PNG. Disponível em: <http://www.tpack.org>. Acceso em: 20 out 2015

KOEHLER, M. J.; MISHRA, P. What is Technological Pedagogical Content Knowledge (TPACK)? Technology and Teacher Education, n. 9, p. 60-70, 2009. DOI: 10.1016/j.compedu.2010.07.009.

KOEHLER, M., MISHRA, P., Y CAIN, W. What Is Technological Pedagogical Content Knowledge (TPACK)? Journal of Education, v. 193, n. 3, p. 13-19, 2013.

INEE. Estudio comparativo de la propuesta curricular de ciencias en la educación obligatoria en México y otros países. México, 2017. Disponível em:

<http://publicaciones.inee.edu.mx/buscadorPub/P1/F/211/P1F211.pdf〉. Acceso em: 03 mar. 2017

RIAEE - Revista Ibero-Americana de Estudos em Educação, Araraquara, v. 13, n. 03, p. 997-1010, jul./set., 2018. E-ISSN: 1982-5587. 
FLORES. F. La enseñanza de la ciencia en la educación básica en México. México: INEE, 2011. Disponível em: <http://publicaciones.inee.edu.mx/buscadorPub/P1/C/227/P1C227.pdf>. Acesso em: 03 mar. 2017.

MORENO TRUJILLO, H.; PINTOR CHÁVEZ, M. M.; GÓMEZ ZERMEÑO, M. G. (2016). Uso de plataformas de libre distribución (LMS) para educación básica. Revista Iberoamericana de Tecnología en Educación y Educación tecnológica, Río de la Plata, n. 15, p. 95-103, jun., 2016.

MACÍAS, A. Recursos Educativos Abiertos para la enseñanza de las ciencias en ambientes de educación básica enriquecidos con tecnología educativa. Dissertaçào (Maestrado em Educaçao), Instituto Tecnológico de Monterrey, México, 18 oct. 2011.

PÓSITO, R. M. El problema de enseñar y aprender Ciencias Naturales en los nuevos ambientes educativos: Diseño de un Gestor de Prácticas de Aprendizaje GPA. Dissertaçào (Maestrado em Tecnología Aplicada) Universidad Nacional de la Plata, Argentina, 19 abr. 2012.

SÁNCHEZ S, R. Plataforma de desarrollo de ciencias integradas 3D ciencias basada en tecnologías de visualización y elementos hapticos para modernizar y apoyar la enseñanza de las ciencias físicas, matemáticas y químicas en la educación básica. CONICYT, Chile, 31 mar. 2009. Disponível em: 〈http://hdl.handle.net/10533/88611〉. Acceso em 09 abr.2017.

SEP. Comunicado 595. 21 de nov. 2016 a. Disponível em:

$<$ https://www.gob.mx/sep/prensa/comunicado-495-fundamentales-ciencia-tecnologia-innovaciony-lectura-en-el-modelo-educativo-la-ciencia-para-todos?idiom=es>. Acceso em: 29 abr. 2017.

SEP. Plan de Estudios 1999. Licenciatura en Educación Secundaria. 3 ed. México: SEP, 2011.

SEP. Ciencias Naturales. Tercer semestre, Licenciatura en Educación Primaria. Plan de Estudios 2012. México: SEP, 2013.

SEP . Acercamiento a las ciencias naturales en preescolar. Tercer semestre, Licenciatura en Educación Preescolar Plan de estudios 2012. México: SEP, 2012.

SEP. Modelo Educativo para la Educación Obligatoria. Educar para la libertad y la creatividad. Ciudad de México. México, 2017. Disponível em:

<https://www.gob.mx/cms/uploads/attachment/file/198738/Modelo_Educativo_para_la_Educacio _n_Obligatoria.pdf>. Acceso em: 29 abr. 2017.

SHULMAN, L. S. Those Who Understand: Knowledge Growth in Teaching. Educational Researcher, v.15, n. 2, p. 4-14.1986. DOI: 10.3102/0013189X015002004

SHULMAN, L. S. Knowledge and teaching: Foundations of the new reform. Harvard Educational Review, n. 57 , v. 1, p. 1-22, 1987.

SHEFFIELD, R.; DOBOZY, E.; GIBSON, D.; MULLANEY, J.; CAMPBELL, C. Teacher education students using TPACK in science: a case of study. Educational Media International, v. 52, n. 3, p. 227-238, 2015. DOI: 10.1080/09523987.2015.1075104.

TONUCCI, F. Con ojos de maestro. Trad. Gladys Kochen. Buenos Aires: Troquel, 1995.

VALVERDE, J.; GARRIDO, M. D. C.; Y FERNÁNDEZ, M. R. Enseñar y aprender con tecnologías: un modelo teórico para las buenas prácticas educativas con TIC. Teoría de La Educación. Educación Y Cultura En La Sociedad de La Información, v. 11, n. 12, p. 03-229, 2010. DOI: $10.1111 /$ j.1467-9620.2006.00684.x.

RIAEE - Revista Ibero-Americana de Estudos em Educação, Araraquara, v. 13, n. 03, p. 997-1010, jul./set., 2018. E-ISSN: 1982-5587. 
YI-FEN, Y.; YING-SHAO, H.; HSIN-KAI, W.; FU-KWUN, H.; TZU-CHIANG, L. Developing and validating technological pedagogical content knowledge-practical (TPACK-practical) through the Delphi survey technique. British Journal Of Educational Technology, v. 45 n. 4, p.707-722, 2014. DOI:10.1111/bjet.12078

ZÁRATE, R.; MORALES, B.; PÉREZ, R. L. La percepción del papel social de la ciencia y la tecnología desde la perspectiva de estudiantes normalistas. Anais... Congreso Iberoamericano Kaanbal, Mérida, Yuc. México. 2016.

\section{Como referenciar este artigo}

COLORADO-AGUILAR, Brenda.; MORALES-GONZÁLEZ, Berenice. Evaluación de los conocimientos tecnológicos, pedagógicos y disciplinares en la enseñanza de la ciencia. Revista Ibero-Americana de Estudos em Educação, Araraquara, v. 13, n. 03, p. 997 1010, jul./set., 2018. E-ISSN: 1982-5587. DOI: 10.21723/riaee.v13.n3.2018.11167

Submetido em: 04/03/2018

Aprovado em: 16/05/2018 